\title{
Virtools Based Development of Computer Experiment
}

\author{
Wei Yuqing, GAO Xing \\ College of Mathematics and Information Technology \\ Hebei Normal University of Science \& Technology \\ Qinghuangdao 066004, China \\ e-mail: hbweiyuqing@yeah.net
}

\author{
GAO Jinghua \\ E and A college \\ Hebei Normal University of Science \& Technology \\ Qinghuangdao 066004, China \\ e-mail: gaojinghua@163.com
}

\begin{abstract}
Virtools is a set of integrated 2D graphic images, 3D models, audio, video, and so a variety of file formats, interactive software itself has a wealth of interactive behavior module, 3D games can be created, virtual experiments simulation objects very strong interaction with the display and other three-dimensional product. This paper presents a virtual experiment based on 3D and Virtools technology, which is built with 3D experiments scenes, making 3D animation; with Virtools data processing, interactive control. The experiments show that this method has the authenticity, interactivity, simple and easy to implement features.
\end{abstract}

\section{Keywords- 3D; Virtools; virtual experiments}

\section{INTRODUCTION (HEADING 1)}

Virtools is developed by the French global interactive three-dimensional development solutions company VIR TOOLS 3D engine has become the Microsoft Xbox recognized system, which is characterized by ease of use, wide application areas. Virtools 3D interactive display is a powerful technology, has the perfect composition: an authoring application, an action engine, a rendering engine, a Web player, a software development kit. Addition to the 3D/VR development platform Virtools Dev five optional modules to suit different applications of virtual reality: the Physics Pack physical attributes, VR Pack, the AI Pack artificial intelligence, Xbox Kit game developers, Virtools Server. Based on Virtools virtual experimental development block diagram including the virtual experimental development platform, a virtual experimental development platform module, modeling software, PC workstations, virtual experiments (VR Content) and browser (Virtools Web Player). The virtual experimental development platform Virtools Dev; virtual experimental development platform module include Virtools Physics Pack, Virtools AI Pack, Virtools VR Pack, Virtools Xbox Kit, Virtools Open CAD, Virtools Server; the main allegations rod of input devices, steering wheel, 3D mouse, 3D gloves location tracking; stereoscopic glasses, stereoscopic screen, headmounted display, analog input device means; to exhibit system includes a three-dimensional theater and display system. As shown in Figure 1. The Virtools itself more than 500 acts of interactive modules (Building Blocks, BB), the user can use these scripts $\mathrm{BB}$ build the appropriate interactive features, interaction design very quickly and easily. The specific operation: the user interface of the software, use the mouse to drag and drop BB BB even in the corresponding object, to form a flow chart of the pre-and post-processing sequence, in order to achieve the visual design of the interactive script, and then completed a virtual interactive system. , Virtools also comes with the SDK (Software Development Kit Software Development Kit) and VSL (Virtools Scripting Language Virtools special script language) through the appropriate API (Application Programming Interface Application Programming) interface, their own writing to create with a specific function behavioral interaction module scripts and applications.

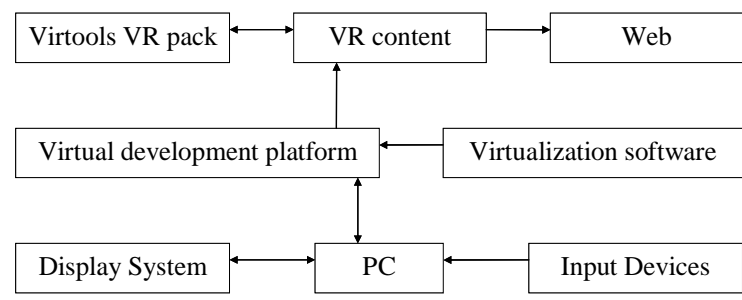

Figure 1. Diagram of virtual experimental development

Virtools also integrates a powerful the Render Engine rendering engine, developers can use the more advanced screen rendering technology.

\section{SigNifICANCE OF VIRTOOLS VIRTUAL EXPERIMENTAL SYSTEM}

Virtual experimental system based inquiry learning theory of constructivism and situated cognition and situated learning theory, under the guidance of the creation of experimental conditions for learners, manufacture cognitive contradiction to stimulate learners' curiosity and develop learners' interest in learning. Virtools technology is to provide a platform to achieve all the functionality of the virtual experimental system. Virtools support for threedimensional modeling software, you can easily achieve the creation of three-dimensional scene, its powerful interactive features, you can realistically simulate the whole process of the experiment, create a problem situation, human-computer interaction, learners immersive experiment. , Virtools powerful rendering engine, support for rich multi-color maps, a virtual experimental system is more colorful in the creation of situations, a strong visual impact and a wealth of experimental conditions, is very effective to stimulate 
learners' interest in learning, greatly improve the learner's motivation to learn.

The system integrated 3ds Max modeling techniques with Virtools interactive technology, the use of their respective advantages complete virtual experimental system function. The experimental apparatus and experimental scene for 3ds Max software virtual experiments, Virtools software for the interactive design of the experiment. Solid Modeling in 3ds Max environment, edited textures with 3ds Max export function (need to install the plug-Max Exporter) scene file (.max) is converted into Virtools supported file formats .nmo output, as shown in figue.2: 3ds Max output .nmo format files .nmo 3ds Max exported file can be imported directly into Virtools in interaction design can also be based on the needs of the experiment, the dynamic load.

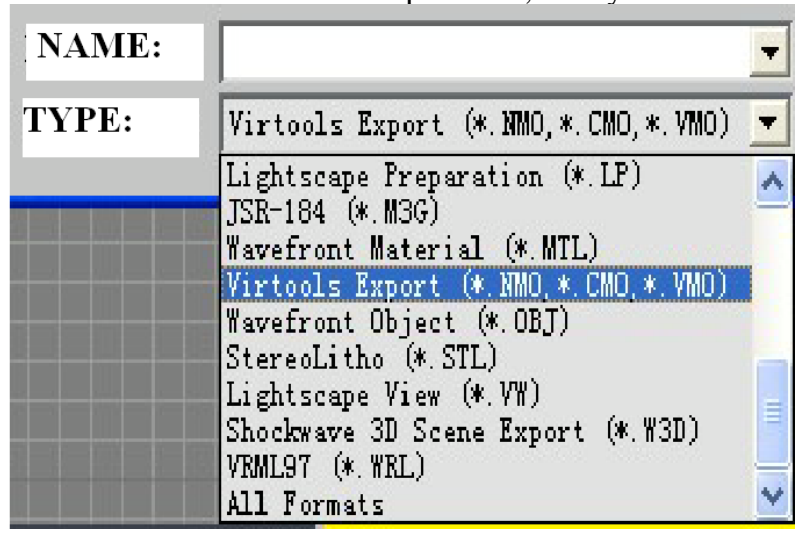

Figure 2. Output .nmo format file of 3DMax

Import 3D models and scenes as part of the Virtools scene selection, rotation, scaling, etc. according to the actual needs of the edit form interactive script was given a series of behavioral interaction module specific interactive features, the ultimate virtual interactive systems (Figure 3).

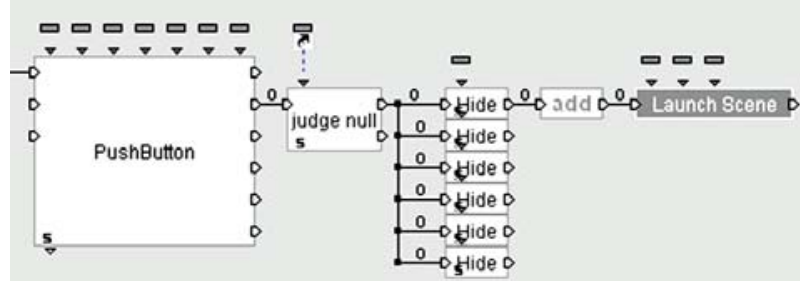

Figure 3. Virtools behavior interaction module interactive script design

\section{ESTABLISH OF EXPERIMENTAL SCENE}

Experimental scene to establish a virtual experimental system requirements as close as possible to the kind of realworld, three-dimensional modeling techniques have been able to model in the virtual scene and scenes produced very realistic and natural, but due to the virtual experimental system in use limit on the speed of the system is running in the process, created during our scenes usually sketched out the scene model then maps to make up the details of the method. Model virtual experimental system generally has a simple, easy to operate, easy to expand features. The virtual experiments need to be the scene of the modeling of the object system (including laboratory building, laboratories, laboratory tables, etc.) and computer hardware experimental devices (including motherboard, CPU, memory, hard disk, host, power supply, optical drive, speakers, monitor, mouse , keyboard, etc.). Since the Virtools software itself does not have the function model creation Therefore, the model is done by 3ds Max 3D modeling software for professional.

In the virtual experiment scene model, the choice of the polygonal modeling method, Virtools itself only supports polygon model. Laboratory building, for example: First of all, we will be building model rectangle, Line spline curve graphics sketched out, linear drawing is completed later modified its local detail in 3ds Max provides edit curve command, the command can modify the three levels of the vertices, lines and curves of the spline curve graphics and editing. Any object by point, line, surface composition, to modify convenience, these objects part independent individual modifications. Details of the modifications are completed, we will have a two-dimensional model is converted to three-dimensional model, modify command panel in 3ds Max stretching, loft command, use these commands can be two-dimensional graphic squeezed into a three-dimensional model, to produce thickness. Model established, we give experimental building model given materials and maps, mostly of photographs on the kind the Photoshop treatment from the texture in order to achieve a realistic effect of this system. Finally, for the scene model branded lighting and rendering output.

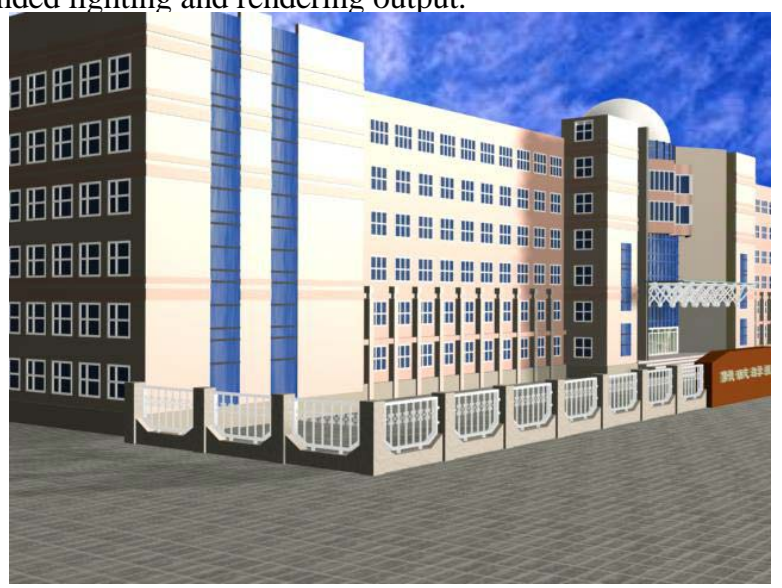

Figure 4. Experimental building part of the scene model

Virtual experimental system taking into account the requirements of the system running speed, scene model should be carried out to establish the principle of simplicity, the model can not produce too fine, and the number of polygonal faces can not be too ideal, less than 800 the number of faces as Virtools can withstand the load range, or to interact over and cause the system to run unstable. Should also be noted that the modeling: the interior angles of the polygon model can not exceed 180 degrees, otherwise it will cause problems on the display; invisible surface (such as the back of the laboratory building) to be deleted to reduce the number of polygon faces; modeling to delete history, further 
optimization process model to go the Virtools after not deform or distort.

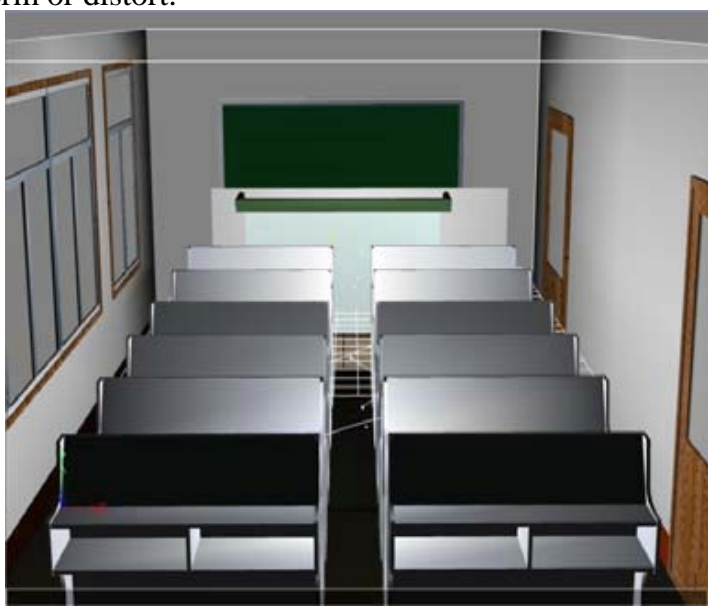

Figure 5. Laboratory scene model

\section{IMPLEMENTATION OF THE INTERACTIVE FEATURES}

We use 3dsMax software develop virtual experiments required components model, Virtools Scripting Language (VSL) component model of interaction behavior control, and behavioral modules use the Virtools development environment in behavior modules (Building Blocks) (Building Blocks) was developed by VC + + NET to achieve, and the whole environment platform running on the Windows XP operating system. Thus the relationship between them as shown in Figure 6:

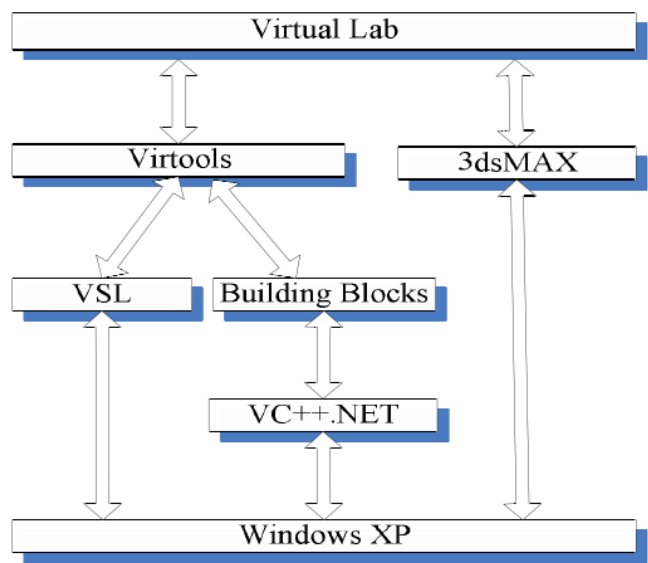

Figure 6. System environment platform

In order to alleviate the burden of the scripting engine to make the system run more smoothly, the system uses the scenes management technology, Virtools offers four modules were encapsulated in the four module named scene a the Level Script (level script) to establish contact between the different scenes, you can easily switch between scenes. Scene management techniques enable the virtual experimental system based on structural, rather than in accordance with the system prescribed order of execution can take care of the experimental needs of the different levels of students, learners can choose according to their needs experimental module learning, reflecting the diversity intelligence theory. Help provide text descriptions to help learners understand before the experiment experimental purposes the main interface including the "enter" experimental interactive buttons and experiments to help explain, "enter" button prompts learners officially entered the experiment, experiment, experiment content, specific experimental procedures and experimental Note matters. The realization of the "enter" button is relatively simple, the procedures described for Figure 6:

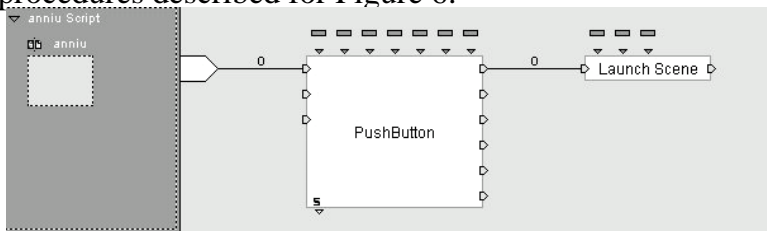

Figure 7. Specific experimental procedures

The Pushbutton activation button, Launch Scene switches to a new scene. Experiment to help design feature: Click "experimental Help button - pop-up experiment illustrates the 2D frame - click on the" OK "button - bounce experimental description of the two-dimensional frame.

\section{CONCLUSION}

In this study, virtual experiment as a teaching aid resources used in computer teaching to try and pave the way to obtain the research results, based on 3ds Max and Virtools technology architecture design and development of virtual experiment method. Development of computer hardware virtual experiments instance, no special requirements on the operation of the system, both hardware and software environment, as long as the installation of the plug-in can run normal; experimental operation, very easy to learn, the operator need only move the mouse experimental operation and scene browsing and keyboard interaction; system updates, very convenient, because the system is based on browser/server mode, the update work need only be performed on the server side; Experiments on the realistic level, the virtual experiment in the development process is to follow a real experiment process and methods of experimental devices and scenes have to do a realistic simulation possible, also try to follow the true mode of operation of the people in an interactive way itself in 3D rendering enhanced intuitive and realistic; experimental arrangements, independent of time and space constraints of the experimental system, and enhance student autonomy; without error attempts during the experiment, allowing students to Consider the platform for the creation of selfinquiry learning laboratory equipment damage and safety hazards to the learner; virtual experimental system can use software as a teaching aid in the classroom teaching, can also be used as a practical hands-on training students the ability to operate the experimental platform Students also can take advantage of virtual experiments previewing and reviewing knowledge. 


\section{ACKNOWLEDGMENT}

This work was financially supported by the Qinhuangdao Soft Science Project of the Science and Technology Bureau (Colleges and Universities Computer Language Examination Reforms and Practice: 2008-1-102).

\section{REFERENCES}

[1] D. Shin, En S. Yoon and S. J. Park, "Web-based interactive virtual laboratory system for unit operations and process systems engineering education,” Computers and Chemical Engineering, vol.12, 2000, pp.1381-1385.

[2] E. R. Jones, “Virtual Reality systems,” Acedemic Press, 1996, pp.4345.

[3] H. Hoyer, A. Joehheim, C. Rhrig and A. Bisehoff, "A Multiuser Virtual-Reality Environment for a Tele-Operated Laboratory,” IEEE Transaetionson Education, vol.52, 2004, pp.590-594.
[4] N. Swamy, "Internet based educational control system lab using netmeeting," IEEE Transaction on education, vol.45, 2002, pp.145151.

[5] M. Renee, W. Jatinder, N. D. G. Mohammed and A. Quaddus, "IT takes a villager:Virtual communities in support of education,” International Jouranal Of Information Management, vol. 20, 2000, pp.473-489.

[6] R. T. Kouzes, J. D. Myers and W. A. Wulf, "Collaboratories:Doing Science on the Internet,” IEEE Computer, vo1.29, no.8, 1996, pp.4046.

[7] R. Laddad, "Aspect-oriented programming will improve quality," Software, IEEE, vo1.20, no.6, November, 2003, pp.90-91.

[8] K. S. Moon and V. Muthukkumarasamy, "Maintaining consistency in distributed network games," IEEE International Conference on Communication, vol.1, November, 2005, pp.374-377.

[9] D. Marshall, D. Roberts and D. Delaney, "Dealing with the Effect of Path Curvature on Consistency of Dead Reckoned Paths in Networked Virtual Environments,” IEEE Virtual Reality. March, 2006, pp. 299-300, 